\title{
An Adaptive Multiple Databases for Rough Set Based Record Deduplication
}

\author{
Ravikanth.M, D.Vasumathi
}

\begin{abstract}
Theoretical. Records duplication is the circumstance wherein unequivocal record is accessible with it's constantly number of copies in the database. To see the records that address a close guaranteed substance, record planning methodology is associated. Today, this is the most signi cant and testing task. The closeness of duplicates in the database will absurdly realize more querry dealing with time and solicitation of extra control resources, etc. To avoid these issues and results, records deduplication strategy is performed.

In this paper, a Rough set based instrument is proposed which e ciently plays out the records planning strategy. These datasets are set up through our records deduplication handling model. Close to the end, algo-rithm produces the dataset gathering, contains exceptional record sections. A short time later, in this paper, investigate results are shown, which are per-formed for standard datasets and execution is poor down
\end{abstract}

Keywords: Duplicate detection, Record linkage, Data deduplication, Data in-tegration, Records matching, Rough sets

\section{INTRODUCTION}

Today, the size of data which is dynamically made from a couple di erent web or querry sources, is inceasing enormously. By far most of which is displayed in unstructured gathering. Thusly, to bring the supportive information from monster volume of data which may be available in sort of cutting edge libraries, e-vaults, online business data, has exhibited as a troublesome issue for the data heads wherever all through the world.

Today, it is a basic test to developed a structure which can play out the going with functionalities:-

\{ Concatenate/arrange distinctive datasets open.

\{ Perform the organizing errand of various available records sets, which may address a comparable substance identified with certified world.

Gigantic proportion of research has been cultivated for records deduplication in a couple of regions like - Data mining, Arti cial knowledge, Databases, Text mining, etc. To ad dress the issue of records deduplication, the courses of action require continuously particular e orts.[1][32] If the inconsequential and unstructured data is accessible in the storage facilities, the outcomes may be (1) progressively computational time and cost (2) corruption in execution (3) all the more learning space dimensionality (4) requires even more master genius cessing power.

To avoid these results, records planning and deduplication is required. The estimation used for records deduplication is

\footnotetext{
Revised Manuscript Received on July 18, 2019.

Ravikanth.M, Research: Department of Computer Science and Engineering' CMR Technical Campus, Medchal, Hyderabad, T.S, India (Email: ravikanthm.cse@gmail.com)

D.Vasumathi, Department of Computer Science and Engineering, JNTUH, Hyderabad, T.S, India (E-mail: rochan44@gmail.com)
}

said to be e cient in case it perceives dynamically number of impersonations through records planning.

\subsection{Related Work}

S.Lawrence et. al. [7] [8] proposed algorithmic techniques eg. adjust partition, express coordinate, etc for references planning from various sources. Kuo-Si Huang et. al. [9] has presented surmise methodologies for preparing the LCS. Surajit Chaudhuri et. al. [10] proposed an e cient facilitate estimation using fleecy method of reasoning, close by that the examinations are performed on the convenient datasets to make the methodology more e ective. Y.Li et. al. [11] have given a method to deal with the substance catagorization issue. Shen et. al. [12] has demonstrated "Soccer(Source Conscious Compiler for Entity Resolution)", a basic procedure to nd impeccable match-ing in record datasets. Diverse syntactic progression game plan segments are used to lterate the site pages having duplicacy. [13] In 2009, Elhadi et. al. [14] have given an improved technique for duplicate acknowledgment in record datasets and site pages.

To address the troublesome circumstance of record planning, Weifeng Su et. al. [15] have presented a solo copy discovery (UDD) procedure. Moises G. et. al. [16] proposed Genetic Programming strategy for records duplicacy detection. In the paper by Madusubram et. al. [17], procedural execution butt-driven ysis is shown for arranged nature of customer web overviews and request. In 2015, Sha Ullah Khan et. al. [18] presented a modi ed "particle swarm improvement" computation for all around updates of general switch unsafe circumstances. In the present client cloud server circumstances, de-duplication on encoded correspondence message is progressing as an enabling example in scienti c arrange. [20] Rodel Miguel et. al. [19] proposed a structure called "HEDup (Homomorphic Encryption Deduplication)" for secure limit circumstance which moreover supports data deduplication.

Execution Comparison of various Learning Methods for Records Deduplication The display comparisions of various signi cant procedures for records instructive files are shortened as table 1 underneath:

\begin{tabular}{|l||l||l|}
\hline \multicolumn{1}{|l|}{ Performance of various Methods for Records Deduplication } \\
\hline & & \\
\hline SVM & 0.926 & \\
\hline OSVM & 0.580 & 0.36 \\
\hline PEBL & 0.902 & 0.42 \\
\hline Christen & 0.886 & 1.42 \\
\hline
\end{tabular}




\subsection{Motivation and Contribution}

In the current circumstance the necessity for records deduplication rises exponentially transcendently in the spaces like databases and data mining. A couple of procedures are proposed in past years for records deduplication. Here, in this paper we will probably introduce a strategy which can play out the system of data deduplication more e ciently. In layout, standard duties of this paper consolidates -

$\{$ this paper shows our proposed technique which performs records deduplica-tion process e ciently. Our proposed procedure utilizes the Roughset as a nstrument in data deduplication figuring model.

\{ frees the customer from the strain of choosing any jumbled streamlining strategy to perform records planning method, which may contains ex-ponential time computational multifaceted nature.

\subsection{Relationship of the paper}

Rest of this paper is overseen as - In portion 2, we have discussed some required essentials. Our proposed estimation is showed up in fragment 3 adjoining analy-sister. Test outcomes are appeared in zone 4 . In piece 5, we have discussed the adjacent appraisal of di erent record organizing structures. Section 6 con-cludes the paper and presented future research headings

\section{EXISTING SYSTEM}

- The Vector Space Model (VSM) is a method for speaking to records through the words that they contain

- It is a standard method in Information Retrieval

- The VSM enables choices to be made about which records are like one another and to catchphrase inquiries

Diagram

- Each report is separated into a word recurrence table

- $\quad$ The tables are called vectors and can be put away as clusters

- A jargon is worked from every one of the words in all records in the framework

- $\quad$ Each record is spoken to as a vector based against the jargon

Model:

- The jargon contains all words utilized

- a, pooch, and, feline, frog

- $\quad$ The jargon should be arranged

- $\quad$ a, and, feline, hound, frog

- Document A

\begin{tabular}{|r|r|r|r|}
\hline $\mathbf{A}$ & dog & and & cat \\
\hline 2 & 1 & 1 & 1 \\
\hline
\end{tabular}

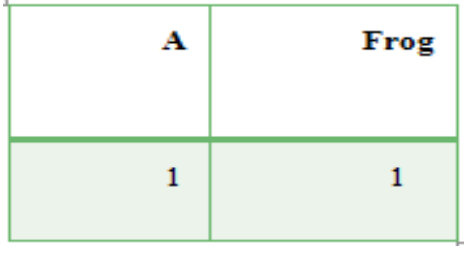

- "A dog and a cat."

- Document B

- "A frog."

- Document A: "A dog and a cat."

\begin{tabular}{|l|l|l|l|l|}
\hline A & and & cat & dog & frog \\
\hline 2 & 1 & 1 & 1 & 0 \\
\hline
\end{tabular}

- $\quad$ Vector: $(2,1,1,1,0)$

- Document B: "A frog."

\begin{tabular}{|c|c|c|c|c|}
\hline A & and & cat & dog & frog \\
\hline 1 & 0 & 0 & 0 & 1 \\
\hline
\end{tabular}

- $\quad$ Vector: $(1,0,0,0,1)$

- Queries can be represented as vectors in the same way as documents:

- $\quad \operatorname{Dog}=(0,0,0,1,0)$

- $\quad$ Frog $=(0,1,0,0,0)$

- $\quad$ Dog and frog $=(0,1,0,0,0)$

- There are many different ways to measure how similar two documents are, or how similar a document is to a query

- The cosine measure is a very common similarity measure

- Using a similarity measure, a set of documents can be compared to a query and the most similar document returned

- For two vectors $\mathrm{d}$ and d' the cosine similarity between $d$ and $d$ ' is given by:

$$
\frac{d \times d^{\prime}}{\left|d \| d^{\prime}\right|}
$$

- Here $\mathrm{d} X \mathrm{~d}$ ' is the vector product of $\mathrm{d}$ and $\mathrm{d}$, calculated by multiplying corresponding frequencies together

- The cosine measure calculates the angle between the vectors in a high-dimensional virtual space

\section{Example}

- Let $\mathrm{d}=(1,0,0,0,1)$ Let $\mathrm{d}=(2,1,1,1,0)$ and $\mathrm{d}^{\prime}=$ $(0,0,0,1,0)$

- $\quad \mathrm{dXd}^{\prime}=2 \mathrm{X} 0+1 \mathrm{X} 0+1 \mathrm{X} 0+1 \mathrm{X} 1+0 \mathrm{X} 0=1$

$$
|\mathrm{d}|=\sqrt{ }\left(2^{2}+1^{2}+1^{2}+1^{2}+0^{2}\right)=\sqrt{ } 7=2.646
$$




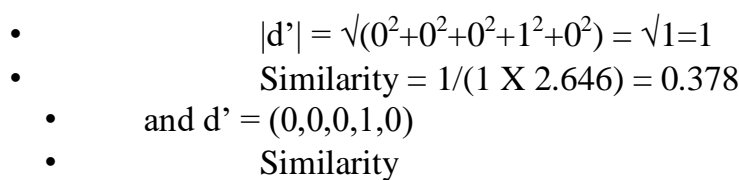

Ranking documents

- A user enters a query

- $\quad$ The query is compared to all documents using a similarity measure

The user is shown the documents in decreasing order of similarity to the query term

\section{PRELIMINARIES}

A few primers required for sRough set based record coordinating and copy identification are as per the following:-

\section{$2.1 \quad$ Rough Sets- $R S$}

Execution of any web records coordinating procedure significantly relies upon the figuring model utilized for this reason. Consider, $\mathrm{S}=(\mathrm{U} ; \mathrm{R})$ be an approxima-tion space, $\mathrm{X}$ is expected here as an idea in that specific space, at that point, lower guess is,

$\mathrm{R}_{\text {lower }} \mathrm{X}=\mathrm{fX} 2 \mathrm{Uj}[\mathrm{x}] \mathrm{Xg}$

upper approximation is constructed as,

$\mathrm{R}_{\text {upper }} \mathrm{X}=\mathrm{fx} 2 \mathrm{Uj}[\mathrm{x}] \backslash \mathrm{X} 6=; \mathrm{g}$

where, $[\mathrm{X}]$ is an equivalence class possessing an element e.

\section{PROPOSED RECORD DEDUPLICATION ALGORITHM BASED ON ROUGH SETS}

This segment displays our proposed calculation for records deduplication utilizing Rough set hypothesis. The general registering model of our proposed calculation is appeared as Figure 1 underneath:-

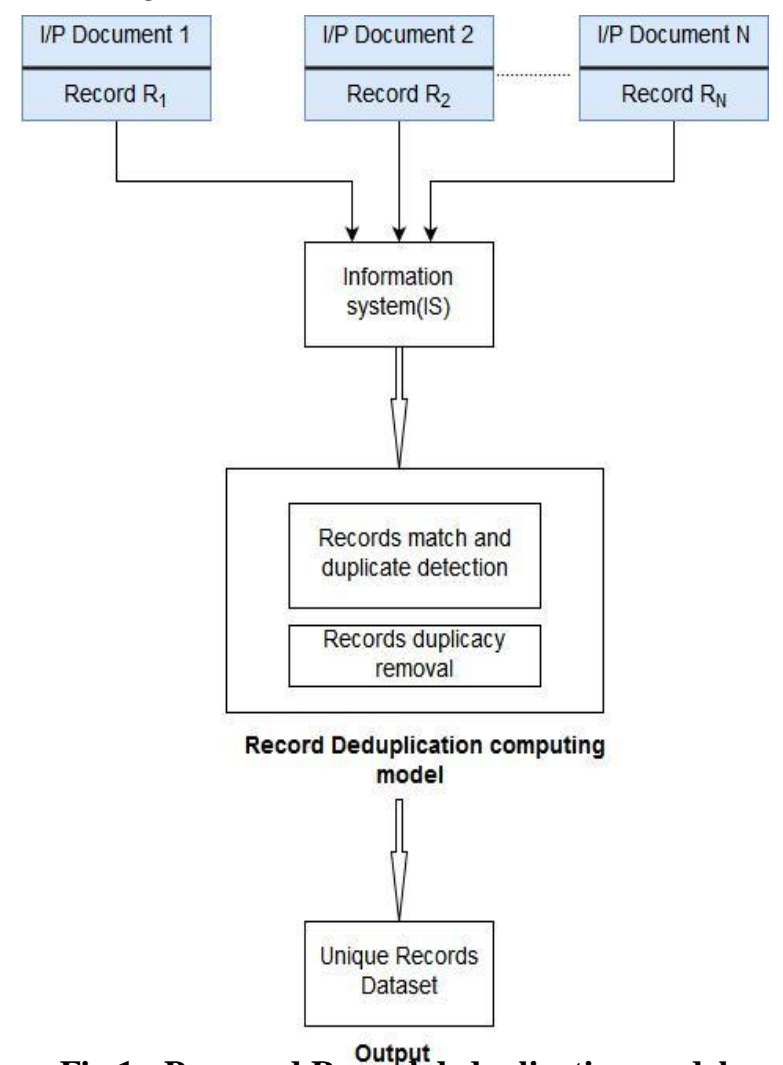

Fig.1 - Proposed Record deduplication model

\subsection{Proposed Algorithm}

The proposed calculation for records deduplication dependent on Rough sets and Log-ical thinking is partitioned into two stages. In stage 1, we have introduced con-struction of the Discernibility Matrix utilizing Rough Sets.

Algorithm 1 Construction of the Discernibility Matrix using RS

1. Input: Di erent dataset documents, where each document consists of several records.

$\mathrm{D}=\mathrm{d}_{1} ; \mathrm{d}_{2} ; \mathrm{d}_{3} ;: .: . ; \mathrm{d}_{\mathrm{n}} ;$ where,

$\mathrm{d}_{\mathrm{i}} 81 \mathrm{i} \mathrm{n}$, represents each $\mathrm{i}^{\text {th }}$ individual document set. BEGIN procedure

2. Concatenate all document sets $\mathrm{d}_{\mathrm{i}} 81 \mathrm{i} \mathrm{n}$ into single set called D. D consists of several individual records $\mathrm{fR}_{1} ; \mathrm{R}_{2}$; $\mathrm{R}_{3} ;:: ; ; \mathrm{R}_{\mathrm{m}} \mathrm{g}$.

3. Consider above records information in the knowledge form of information table (IS).

Information System - An information structure in RST is addressed as pair (U; A), where -

$\mathrm{U}$ : demonstrates non-void nite set of things.

A: demonstrates non-void nite set of properties.

4. In our information table, each row represents individual record.

$\mathrm{R}_{\mathrm{i}}=\mathrm{fR}_{1} ; \mathrm{R}_{2} ; \mathrm{R}_{3} ;::: ; ; \mathrm{R}_{\mathrm{m}} \mathrm{g}$; here assume, $\mathrm{m}$ di erent records are present. each column represents di erent attribute vectors

$\mathrm{V}=\mathrm{fv}_{1} ; \mathrm{v}_{2} ; \mathrm{v}_{3} ;::: ; \mathrm{v}_{\mathrm{k}} \mathrm{g}$; here assume, $\mathrm{k}$ di erent vectors are present in IS.

5. Compute Discernibility matrix for given IS.

Discernibility matrix in RST - "A discernibility matrix of an information table

$\mathrm{I}=(\mathrm{U} ; \mathrm{A})$ is a symmetric $\mathrm{jUj} \mathrm{X} \mathrm{jUj}$ matrix with it's entries as- $\mathrm{c}_{\mathrm{ij}}=\mathrm{fa} 2 \mathrm{Aja}\left(\mathrm{x}_{\mathrm{i}}\right) 6=\mathrm{a}\left(\mathrm{x}_{\mathrm{j}}\right) \mathrm{g} ; \mathrm{i} ; \mathrm{j}=1 ;::: ; \mathrm{jUj}$

Individual $\mathrm{c}_{\mathrm{ij}}$ consists of those features, who o ers the di erence between objects $i$ and $j "$.

In phase 2, we have displayed records deduplication utilizing Rough Set hypothesis and Logical thinking.

Algorithm 2 Records deduplication using RS and Logical reasoning

1. Draw Perceptibility table in which all conceivable record set sets are included, repre-sentation of them is as individual lines and record qualities go about as sections.

2. Perform ordering in the Perceptibility table utilizing sensible suggestions [(entries either $\mathrm{T}$ or 1 ) or (sections either $\mathrm{F}$ or 0 )].

3. Do logical reasoning of sections for each line in listed detectable quality table. Perform coherent 'OR' activities for comparing passages in each row.

4. Store result entries in new column vector ${ }^{0} \mathrm{DV}^{0}$

5. Search for logical entry as $\mathrm{F}$ or 0 , present in ${ }^{0} \mathrm{DV}^{0}$.

6. The row record pairs corresponding to $\mathrm{F}$ or 0 entries can be identi ed as duplicate record pairs.

Published By: 
7. Remove those identified records from IS (Information table). Dimensionality of records set is decreased after deduplication process.

END procedure

\subsection{Analysis of Proposed algorithm}

Our proposed calculation for records deduplication includes the development of detectability framework. While building detectability grid, each record ob-ject case column includes jmj comparisions. Where, jmj speaks to the complete number of article instances of a record. So the quantity of examinations required as - jmj. Along these lines, unpredictability for development of detectability lattice is $\mathrm{O}(\mathrm{jmjjmj})$.

\subsection{Proposed Method}

Rs Based De-Duplication

Algorithm 1 Construction of the Discernibility Matrix using RS:

1. imput : 'Different dataset documents, where each document consists of several records.

$D=d_{1}, d_{2}, \ldots, d_{n} ;$ Where, $d_{i} \forall 1 \leq i \leq n$, represents each $i^{\text {th }}$ individual document set. BEGIN procedire

2. Concatenate all document $d_{i} \forall 1 \leq i \leq n$, sets into single set called $D$. D Consists of several individual records $\left\{R_{1}, R_{2}, R_{3}, \ldots, R_{m}\right\}$.

3. Consider above records information in the knowledge form of information table (IS).

Information System :An information system in RST is represented as pair (U,A).

Where, $U$ : denotes non-empty finite set of objects.

A : denotes non-empty finite set of attributes.

4. IIn our minormation rade, eacn row representrs manvinual recora.

$R_{i}=\left\{R_{1}, R_{2}, R_{3}, \ldots, R_{m}\right\} ;$ here assume, $m$ different records are present.

Each columnn reppresents different attribute vectors:

$V=\left\{v_{1}, v_{2}, V_{3}, \ldots, v_{k}\right\} ;$ Here assume, $k$ different vectors are present in IS.

5. Compute Discernibility matrix for given IS.

Discernibility matrix in RST - A discernibility matrix of an information table $I=(U, A)$ is a

symmetric $|U| X|U|$ matrix with its entries as:

$$
c_{i j}=\left\{a \in A \mid a\left(x_{i}\right) \neq a\left(x_{j}\right)\right\} ; i, j=1, \ldots,|U|
$$

Individual $c_{i j}$ consists of those features, who offers the difference between objects $i$ and $j$.
Algorithm 2 Records deduplication using RS and Logical reasoning.

1. Draw Perceptibility table in which all possible record set pairs are involved, representation of them is as individual rows and record attributes act as columns.

2. Perform indexing in the Perceptiblity table using logical propositions [(entries either T or 1 ) or (entries either $F$ or 0 )]

3. Do logical reasoning of entries for each row in indexed perceptibility table. Perform logical 'OR' operations for corresponding entries in each row.

4. Store result entries in new column vector' $' D V^{\prime}$

5. Search for logical entry as $F$ or 0 , present in ' $D V^{\prime}$.

6. The row record pairs corresponding to $F$ or 0 entries can be identified as duplicate record pairs.

7. Remove those identified records from IS (Information table). Dimensionality of records set is reduced after deduplication process.

\section{END procedure}

These datasets are prepared through our records deduplication registering model. Toward the end, calculation creates the dataset gathering, comprises of special record passages.

- This proposed technique uses harsh set as an instrument and effectively liberates the client from the strain of deciding any entangled advancement system to perform records coordinating procedure, which may comprises of exponential time computational intricacy.

- $\quad$ Our proposed calculation for records deduplication includes the development of perceptibility network. While building perceptibility grid, each record article case line includes $|\mathrm{m}|$ examinations.

- Where, $|\mathrm{m}|$ speaks to the absolute number of item instances of a record or the element of the perceptibility lattice. So absolute number of correlations required are $|\mathrm{m}| \times|\mathrm{m}|$. Along these lines, intricacy for development of perceptibility network is $\mathrm{O}(|\mathrm{m} \| \mathrm{m}|)$.

Execution EVALUATION

- We have played out our investigations utilizing bigger datasets eg. Reuters, Amazon book audits, Hotel surveys and so forth. Reuters-21578 is a standard corpus for doing the assignment of records coordinating, de-duplication and further complete the arrangement.

- The Reuters-21578 immense reports accumulation, showed up on Reuters newswire in 1987, comprises of 21578 English archives alongside 135 classes.

- We utilize the "ModApte" split rendition of Reuters 21578 and pick the seven most successive Reuters classifications without duplication as our calculation corpus.

- Later we broke down the exactness of the trials and normal execution time required for various arrangement machines. 
- Our test results reasons that our methodology is having relatively lesser computational time and plays out the records coordinating and de-duplication process all the more effectively.

The stepwise component strategy is as underneath:-

1. All record sets are connected into single set called D. D comprises of a few individual record $\{\mathrm{R} 1, \mathrm{R} 2, \ldots, \mathrm{Rm}\}$. At that point, above records data is spoken to in the learning type of data table (IS). In this built data table, each line speaks to individual record and every section speaks to various property vectors.

2. Algorithmic execution begins with the objective of records coordinate, copy discovery and duplicacy expulsion. The calculation of detectability lattice for given is performed.

3. Later in this procedure, push record sets are recognized as copy record sets. Toward the end, expulsion of those recognized records from is finished. After the total procedure, record duplicacy expulsion is done and further records characterization can be performed proficiently.

4. We have performed explores by taking fluctuating number of dispersed

records-set database areas. Every database comprises of different thing sets. Reuters-21578 standard dataset is taken as contribution here. For each situation, the comparing special record-sets are mined and streamlining execution is broke down.

- In underneath table (I) to (iv), we have considered the no. of various circulated record sets databases

- $\mathrm{n}[\mathrm{RS}]=3,4,5$, and 6

- $\quad \mathrm{N}(\mathrm{I})$ : no. of examples

- In tables, we have investigated the execution time taken during the time spent record-sets coordinating, deduplication. For this, the standard Reuters-21578 dataset [1] is used.

- We have performed tests by taking fluctuating number of disseminated record-set areas. The outcomes are exhibited in tables (I) to (iv).

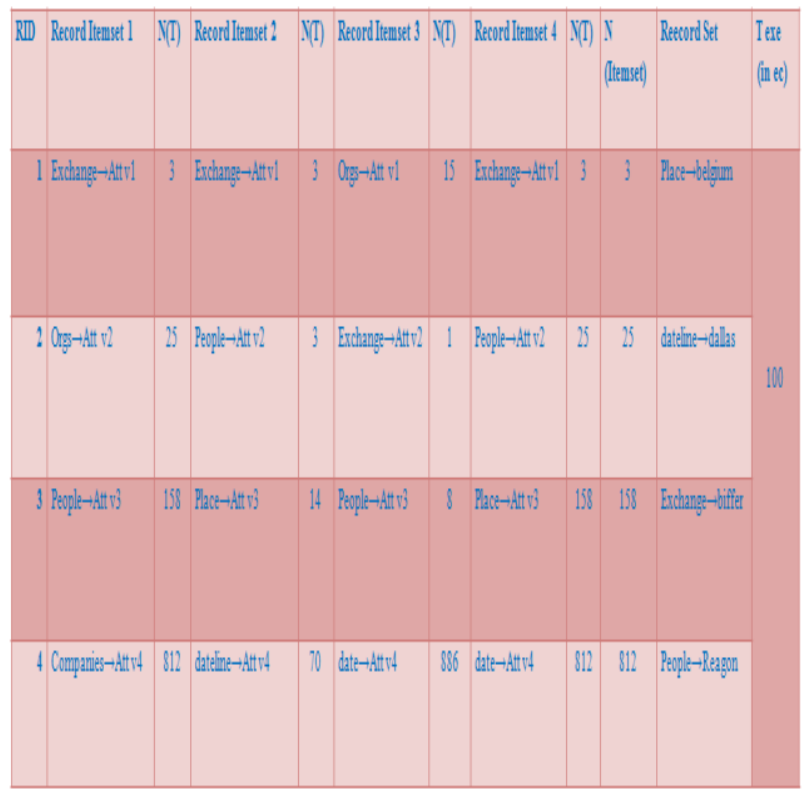

Table 1:RS Based de-duplication

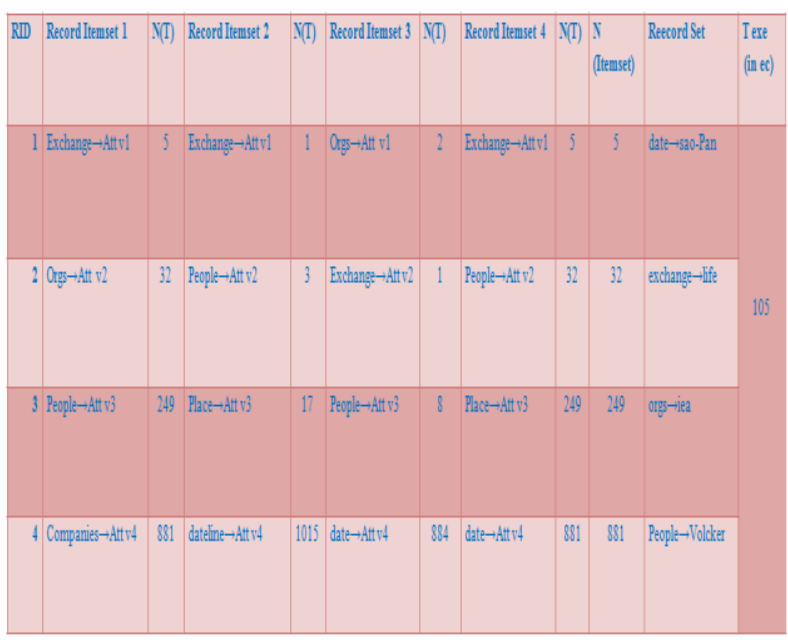

Table 2:RS Based de-duplication

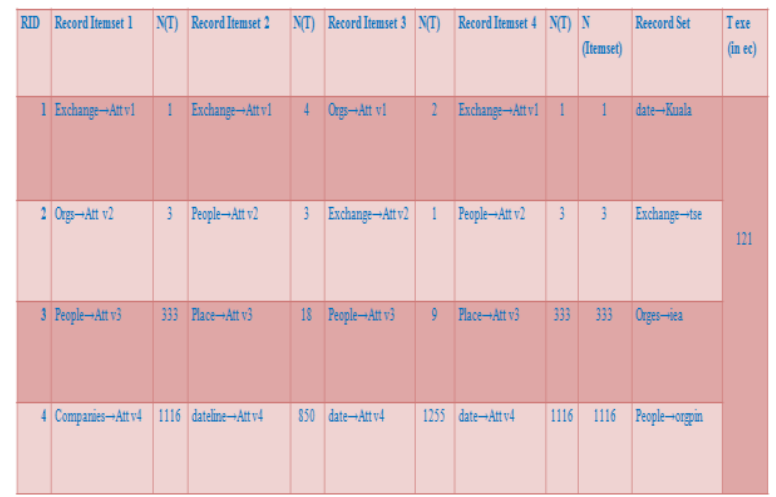

Table 3:RS Based de-duplication

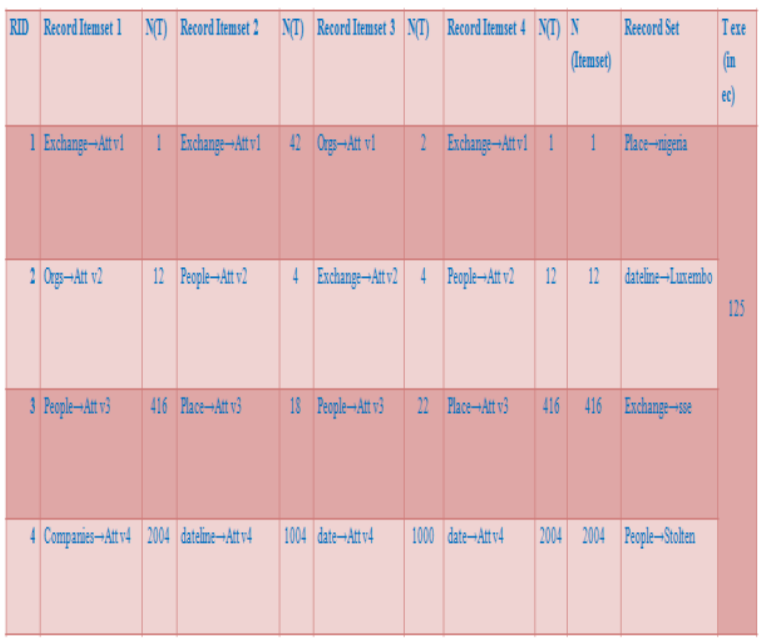

Table 4:RS Based de-duplication

The execution performance analysis graphs for varying number of different distributed record sets databases are given as below:- 


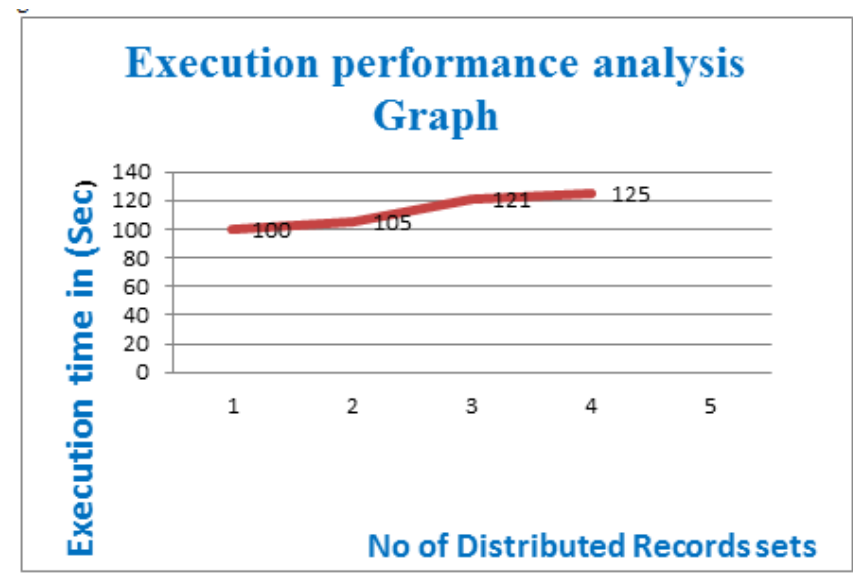

- Graph show the execution time for records matching and deduplication process for varying cases of number of distributed record-set databases

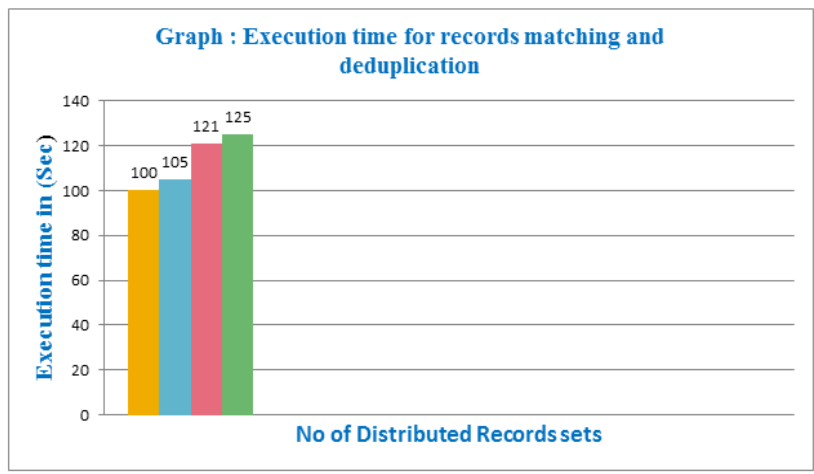

\section{EXPERIMENTAL RESULTS}

The software and hardware speci cations, reproduction condition arrangement and strategy is nitty gritty as underneath:-

\subsection{System Speci cations}

Our framework speci cations are as beneath:-

\{ Software Speci cations: OS - Ubuntu 16.04 LTS, 64 bit, Java variant - '1.8.0 111'.

\{ Hardware Speci cations:RAM size - 4 GB, Processor Intel center i3 4030U CPU @ 1:90GHz 4

\subsection{Input and Setup}

In our test, we have assembled di erent dataset files, where each report involves a couple of records. These reports are as reference nuances of various research papers, assembled from di erent sources. Each docu-ment, containing reference records constrains it's properties as Title, Author nuances, Journal/meeting nuances, Vol. number, Month, Year, etc. By then all record sets di $81 \mathrm{I} \mathrm{n}$ are associated into single set called D. D includes sev-eral individual records fR1; R2; R3; ::::; Rmg. By then, above records information is addressed in the learning sort of information table (IS). In this manufactured information table, each line addresses individual record and each fragment repre-sents di erent trademark vectors. In our investigation we have assembled 410 records from various web sources. Nearby this we have pondered 6 attributes. Along these lines, our data IS table size is 4106 .

\subsection{Procedure}

\{ Algorithmic execution starts with the goal of records arrange, duplicate location and duplicacy removal. In the framework, our data is 4106 estimation size of IS containing various records. The figuring of perceptibility network for given is performed. By then in the wake of outline recognizable quality table, containing record set sets, requesting is performed using reliable recommendations.

$\{$ Later in this system, push record sets are identi ed as duplicate record sets. Close to the end, departure of those identi ed records from is done. After the absolute methodology, record duplicacy ejection is done and the proposed RDCM model gives the yield as Records Data set having all record entries as uncommon.

$\{$ The end results are shown as table 2 below:-

Table-2

\begin{tabular}{|l||l|l||l|}
\hline \multicolumn{4}{|c|}{ Table for execution time } \\
\hline $\begin{array}{l}\text { Records dataset (IS } \\
\text { size) }\end{array}$ & $\begin{array}{l}\text { Duplicate } \\
\text { records }\end{array}$ & $\begin{array}{l}\text { Unique } \\
\text { records }\end{array}$ & $\begin{array}{l}\text { Avg. execution } \\
\text { time (in sec) }\end{array}$ \\
\hline 4106 & 99 & 311 & 0.0678 \\
\hline 5156 & 132 & 383 & 0.0912 \\
\hline 6106 & 169 & 441 & 0.0989 \\
\hline 7506 & 201 & 549 & 0.1203 \\
\hline 8906 & 239 & 651 & 0.2376 \\
\hline 10006 & 312 & 688 & 0.3059 \\
\hline
\end{tabular}

\{ The execution performance analysis for di erent dimensional size Record datasets is represented as graphs below :-

Buahib patimast ent

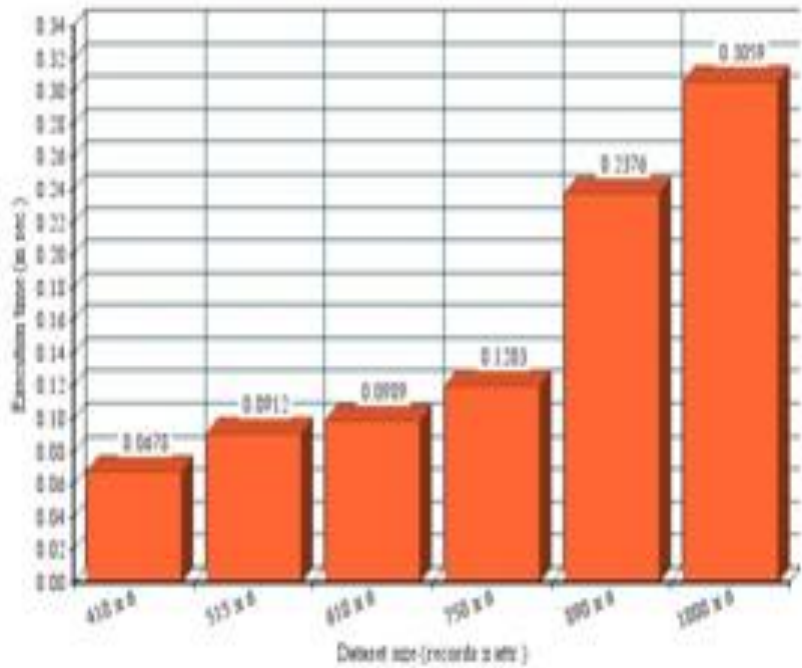

Performance graph.1 
Figure 2: Comparing the three different methods of creating Committees

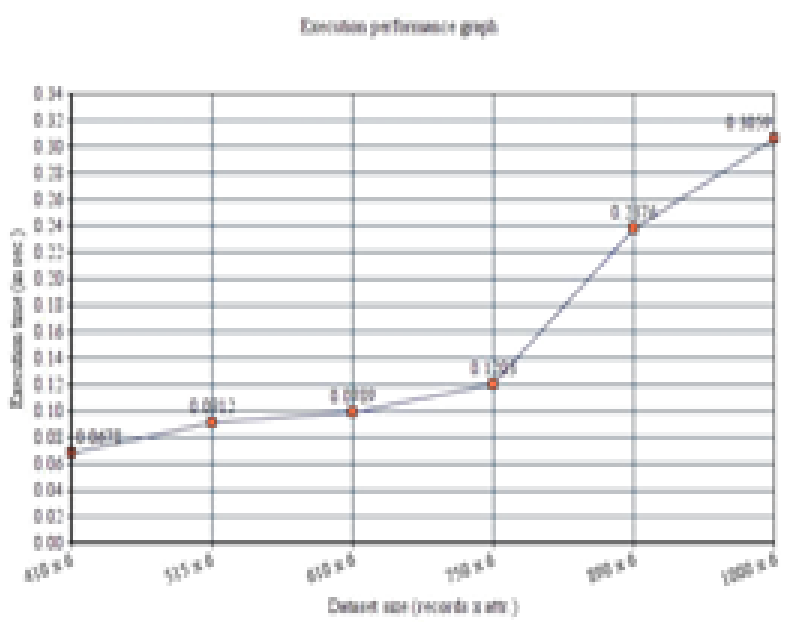

Performance graph.2

\section{COMPARATIVE EXAMINATION}

The crucial focal points of RS speculation which is being utilized in our proposed system model for records deduplication are as underneath -

\{ RS based records deduplication approach utilizes later advanced computa-tional gadget for instance Unpleasant sets for variable's decision or to nd the Reduct.

\{ Any kind of additional estimations for instance probabilistic repeat of data or other explicit irrefutable information.

\{ It performs dimesionality decline of the records instructive file learning by clearing irrelavent attributes.

As diverge from the avg. execution time taken by various techniques (which are total marized in table 1), our proposed algorithmic model for Records deduplication using RS and Logical reasoning takes less avg. execution time (dense in table 2).

Looking at the three di_erent strategies for making council

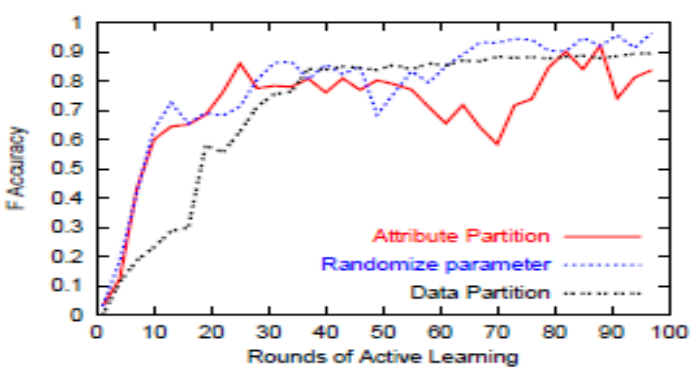

(a) Bibliography data

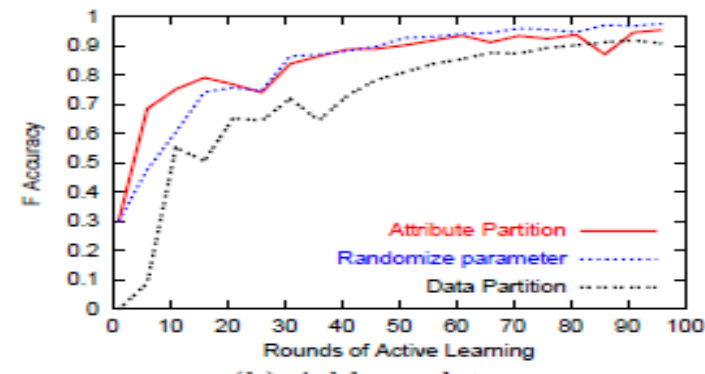

(b) Address data

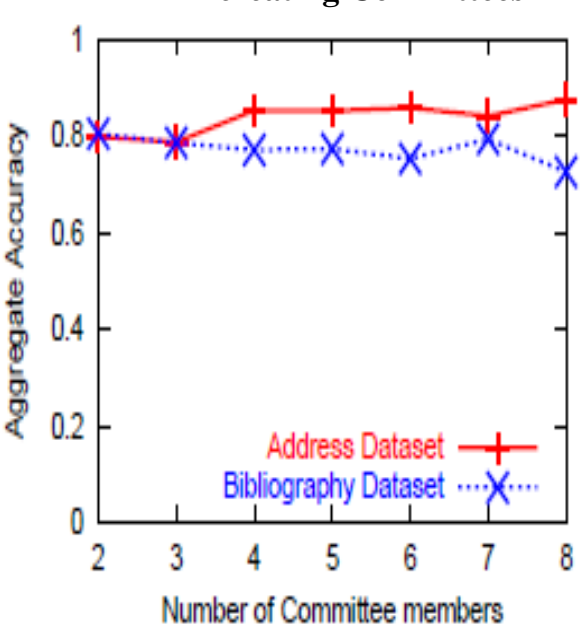

Figure 3: Change in aggregate accuracy with varying number of committee members

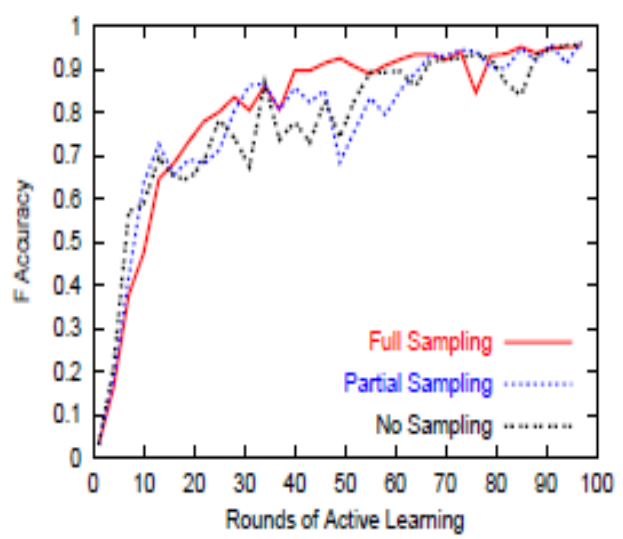

(a) Bibliography data: Decision tree

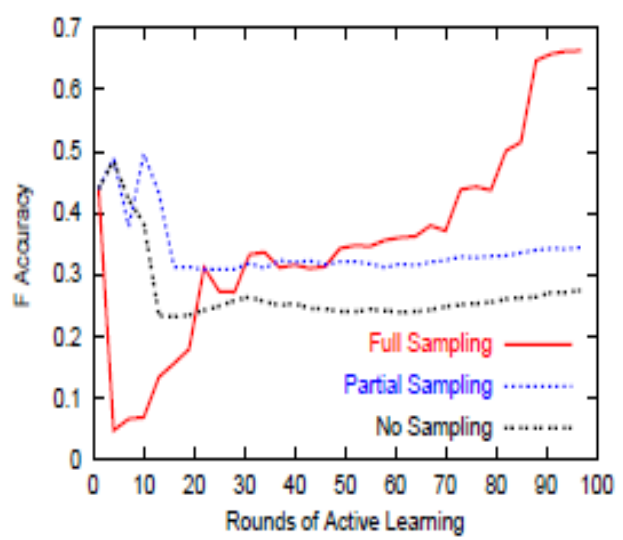

(b) Bibliography data: Naive Bayes

Figure 4: Comparing di_erent sampling schemes for incorporating representative instances 


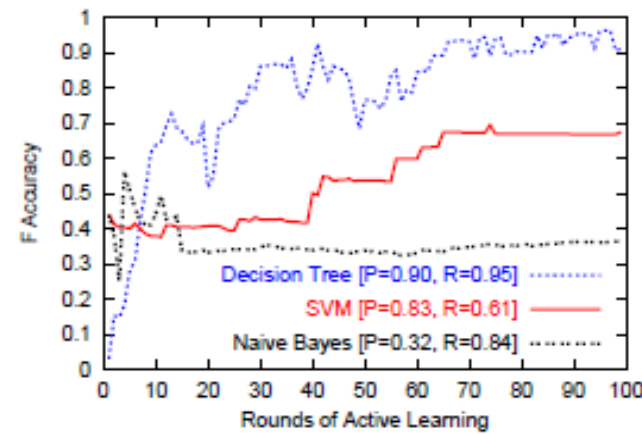

(a) Bibliography data

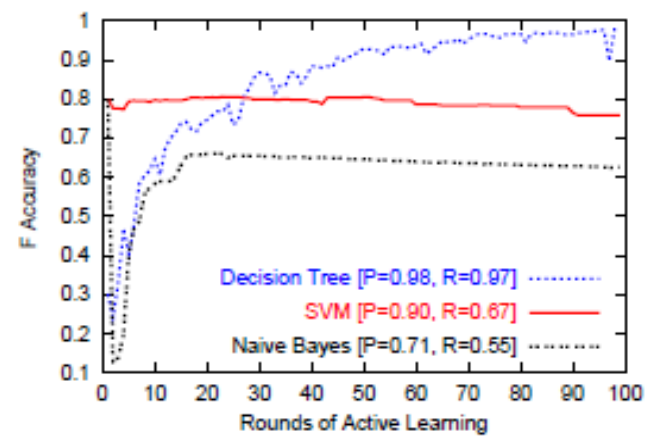

(b) Address data

\section{Figure 5: Comparing performance of different classification methods with active learning.}

\section{CONCLUSION}

This paper shows our proposed strategy which performs records deduplication process e ciently. Our proposed methodology utilizes the Roughset as a gadget in data deduplication figuring model. Investigation results exhibits that our system is having also lesser computational time and plays out the records dedupication process more e ciently. In future we will play out our examinations using greater datasets eg. Reuters, Cora, Amazon book reviews, Hotel, Movie reviews datasets, etc in our Record deduplication figuring framework. Later we will separate the precision of the preliminaries and ordinary execution time required for di erent con guration machines.

\section{REFERENCES}

1. M. Wheatley, "Activity Clean Data", CIO Asia Magazine, http:==www.cio-asia.com, Aug 2004.

2. N. Koudas, S. Sarawagi, and D. Srivastava, "Record Linkage: Similarity Measures and Algorithms", Proc. ACM SIGMOD International Conference on Management of Data, pp. 802-803, 2006.

3. I. Bhattacharya and L. Getoor, "Iterative Record Linkage for Cleaning and Inte-gration", Proc. ninth ACM SIGMOD Workshop Research Issues in Data Mining and Knowledge Discovery, pp. 11-18, 2004.

4. I.P. Fellegi and A.B. Sunter, "A Theory for Record Linkage", J. Am. Measurable Association, vol. 66, no. 1, pp. 1183-1210, 1969.

5. R. Chime and F. Dravis, "Would you say you is Data Dirty? also, Does that Matter?", Accenture Whiter Paper, http:==www.accenture.com, 2006.

6. Pawlak, Z. (1982). Harsh sets. Global Journal of Computer and Information Sciences, 11(5), 341356.

7. S. Lawrence, C.L. Giles, and K.D. Bollacker, "Selfgoverning Citation Matching", Proc. Third Int'l Conf. Self-sufficient Agents, pp. 392-393, 1999.
8. S. Lawrence, L. Giles, and K. Bollacker, "Advanced Libraries and Autonomous Cita-tion Indexing", Computer, vol. 32, no. 6, pp. 67-71, June 1999.

9. Kuo-Si Huang, Chang-Biau Yang, and Kuo-Tsung Tseng, "Quick Algorithms for Finding the Common Subsequence of Multiple Sequences", National Science Coun-cil of the Republic of China, NSC-90-2213-E-110015 .

10. H. Galhardas, D. Florescu, D. Shasha, E. Simon, and C. Saita. Decisive information cleaning: Language, model and calculations. In Proc. of the 27th Int'l Conference on Very Large Databases (VLDB), pages 307\{316, Rome,Italy, 2001.

11. L. Gravano, Panagiotis, and H. V. Jagadish. Inexact string participates in a database (nearly) for nothing. In Proc. of the 27th Int'l Conference on Very Large Databases (VLDB), Rome,Italy, 2001.

12. M. A. Hernandez and S. J. Stolfo. Genuine information is grimy: Data purifying and the union/cleanse issue. Information Mining and Knowledge Discovery, 2(1):9\{37, 1998.

13. J. Hylton. Recognizing and consolidating related bibliographic records. Ace's proposition, MIT, 1996.

14. V. S. Iyengar, C. Apte, and T. Zhang. Dynamic getting the hang of utilizing versatile resampling. In $\mathrm{R}$. Ramakrishnan, S. Stolfo, R. Bayardo, and I. Parsa, editors, Proceedinmgs of the sixth ACM SIGKDD International Conference on Knowledge Discovery and Data Mining (KDD-00), pages 91\{98, N. Y., Aug. $20\{23$ 2000. ACM Press.

15. R. Kohavi, D. Sommer_eld, and J. Dougherty. Information mining utilizing MLC++: An AI library in $\mathrm{C}++$. In Tools with Arti_cial Intelligence, pages $234\{245$. IEEE Computer Society Press, accessible from http://www.sgi.com/tech/mlc/, 1996.

16. S. Lawrence, C. L. Giles, and K. Bollacker. Advanced libraries and independent reference ordering. IEEE Computer, 32(6):67\{71, 1999.

17. R. Liere and P. Tadepalli. Dynamic learning with boards for content arrangement. In Proceedings of AAAI-97, fourteenth Conference of the American Association for Arti_cial Intelligence, pages 591\{596, Providence, US, 1997. AAAI Press, Menlo Park, US.

18. A. McCallum, K. Nigam, J. Reed, J. Rennie, and K Seymore. Cora: Computer science research paper web index. http://cora.whizbang.com/, 2000.

19. A. McCallum, K. Nigam, and L. H. Ungar. E_cient grouping of high-dimensional informational collections with application to reference coordinating. In Knowledge Discovery and Data Mining, pages 169\{178, 2000.

20. A. K. McCallum and K. Nigam. Utilizing EM in poolbased dynamic learning for content classi_cation. In J. W. Shavlik, editorial manager, Proceedings of ICML-98, fifteenth International Conference on Machine Learning, pages 350\{358, Madison, US, 1998. Morgan Kaufmann Publishers, San Francisco, US.

21. T. Mitchell. AI. McGraw-Hill, 1997.

22. A. E. Monge and C. P. Elkan. The _eld coordinating issue: Algorithms and applications. In Proceedings of the Second International Conference on Knowledge Discovery and Data Mining (KDD-96), 1996.

23. G. Navarro. A guided visit to inexact string coordinating. ACM Computing Surveys, 33(1):31\{88, 2001

24. J. R. Quinlan. C4.5: Programs for Machine Learning. Morgan Kaufman, 1993. programming accessible from http:/www.cse.unsw.edu.au/ quinlan/c4.5r8.tar.gz.

25. V. Raman and J. M. Hellerstein. Potters wheel: An

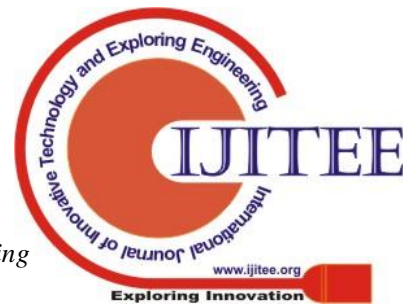


intelligent information cleaning framework. In Proc. of the 27th Int'l Conference on Very Large Databases (VLDB), pages 307 \{316, Rome, Italy, 2001.

26. S. Sarawagi, editorial manager. IEEE Data Engineering uncommon issue on Data Cleaning. http://www.research. microsoft.com/look into/db/debull/A00dec/issue. htm, December 2000.

27. G. Schohn and D. Cohn. Toning it down would be best: Active learning with help vector machines. In Proc. seventeenth International Conf. on Machine Learning, pages 839 \{ 846. Morgan Kaufmann, San Francisco, CA, 2000.

28. H. S. Seung, M. Opper, and H. Sompolinsky. Question by board. In Computational Learing Theory, pages $287\{294,1992$.

29. S. Toney. Cleanup and deduplication of a global bibliographic database. Data Technology and libraries, 11(1):19 \{ 28, 1992.

30. S. Tong and D. Koller. Bolster vector machine dynamic learning with applications to content classi_cation. Diary of Machine Learning Research, 2:45\{66, Nov. 2001.

31. W. E. Winkler. Coordinating and record linkage. In B. G. C. et al, proofreader, Business Survey Methods, pages 355 \{384. New York: J. Wiley, 1995. accessible from http://www.census.gov/.

32. W. E. Winkler. The condition of record linkage and flow inquire about issues. RR99/04, http://www.census.gov/srd/papers/pdf/rr99-04.pdf, 1999.

33. B. Zadrozny and C. Elkan. Learning and settling on choices when expenses and probabilities are both obscure. In Proceedings of the Seventh International Conference on Knowledge Discovery and Data Mining (KDD), 2001.

34. T. Zhang and F. J. Oles. A likelihood investigation on the estimation of unlabeled information for classi_cation issues. In Proc. seventeenth International Conf. on Machine Learning, pages 1191\{1198. Morgan Kaufmann, San Francisco, CA, 2000. 